\section{(6) OPEN ACCESS}

\title{
Cost-effectiveness of implantable cardiac devices in patients with systolic heart failure
}

\author{
Stuart Mealing, ${ }^{1}$ Beth Woods, ${ }^{2}$ Neil Hawkins, ${ }^{1}$ Martin R Cowie, ${ }^{3}$ \\ Christopher J Plummer, ${ }^{4}$ William T Abraham, ${ }^{5}$ John F Beshai, ${ }^{6}$ Helmut Klein, ${ }^{7}$ \\ Mark Sculpher ${ }^{1,2}$
}

\begin{abstract}
- Additional material is published online only. To view please visit the journal online (http://dx.doi.org/10.1136/ heartjnl-2015-308883).

${ }^{1}$ ICON Health Economics, ICON Clinical Research (UK) Ltd., Abingdon, UK

${ }^{2}$ Centre for Health Economics, University of York, York, UK

${ }^{3}$ National Heart \& Lung Institute, Imperial College London (Royal Brompton Hospital), London, UK ${ }^{4}$ Cardiothoracic Directorate, Freeman Hospital, Newcastle upon Tyne, UK ${ }^{5}$ Department of Internal Medicine, Ohio State University Medical Centre, Columbus, Ohio, USA

${ }^{6}$ Department of Cardiovascular Diseases Mayo Clinic, Phoenix, Arizona, USA

${ }^{7}$ University of Rochester Medical Centre, University of Rochester, New York, USA
\end{abstract}

\section{Correspondence to}

Stuart Mealing, Divisional Principal, European Head Health Economics, ICON Clinical Research (UK) Ltd. West Way, Botley OX2 0JJ, UK; stuart.mealing@iconplc.com

Received 26 October 2015 Revised 17 May 2016

Accepted 18 May 2016 Published Online First 13 July 2016

\begin{abstract}
Objective To evaluate the cost-effectiveness of implantable cardioverter defibrillators (ICDs), cardiac resynchronisation therapy pacemakers (CRT-PS) and combination therapy (CRT-D) in patients with heart failure with reduced ejection fraction based on a range of clinical characteristics.
\end{abstract}

Methods Individual patient data from 13 randomised trials were used to inform a decision analytical model. A series of regression equations were used to predict baseline all-cause mortality, hospitalisation rates and health-related quality of life and device-related treatment effects. Clinical variables used in these equations were age, QRS duration, New York Heart Association (NYHA) class, ischaemic aetiology and left bundle branch block (LBBB). A UK National Health Service perspective and a lifetime time horizon were used. Benefits were expressed as quality-adjusted life-years (QALYs). Results were reported for 24 subgroups based on LBBB status, QRS duration and NYHA class.

Results At a threshold of $£ 30000$ per QALY gained, CRT-D was cost-effective in 10 of the 24 subgroups including all LBBB morphology patients with NYHA I/II/ III. ICD is cost-effective for all non-NYHA IV patients with QRS duration $<120 \mathrm{~ms}$ and for NYHA I/II non-LBBB morphology patients with QRS duration between $120 \mathrm{~ms}$ and $149 \mathrm{~ms}$. CRT-P was also cost-effective in all NYHA III/IV patients with QRS duration >120 ms. Device therapy is cost-effective in most patient groups with LBBB at a threshold of $£ 20000$ per QALY gained. Results were robust to altering key model parameters. Conclusions At a threshold of $£ 30000$ per QALY gained, CRT-D is cost-effective in a far wider group than previously recommended in the UK. In some subgroups ICD and CRT-P remain the cost-effective choice.

\section{INTRODUCTION}

In addition to guideline directed medical therapy, implantable cardiac devices have an established role in the treatment of heart failure with reduced ejection fraction (HFrEF). International clinical guidelines ${ }^{1-3}$ make recommendations for implantaCrossMark ble cardioverter defibrillators (ICDs), cardiac resynchronisation therapy pacemakers (CRT-P) and the combined device, CRT-D, based on the presence of specific patient characteristics, recognising that the extent of clinical benefit associated with these devices varies across subgroups within the broader population of patients with HRrEF.
Healthcare systems internationally are seeking evidence on the value for money of medical interventions, and ICD and CRT have been the subject of a number of economic evaluations ${ }^{4-9}$ and health technology assessments (HTAs). ${ }^{10-12}$ In general, the conclusion has been that ICD and CRT are costeffective when compared with medical therapy, and that CRT-D is marginally cost-effective when compared with stand-alone CRT-P.

Clinical guidelines on ICD/CRT in HF make recommendations based on a range of clinical parameters. These include New York Heart Association (NYHA) class, left ventricular ejection fraction (LVEF), ischaemic aetiology status, QRS duration, and presence or absence of either atrial fibrillation or left bundle branch block (LBBB). ${ }^{2}{ }^{3}$ Guidance issued by HTA organisations such as the National Institute for Health and Care Excellence $(\mathrm{NICE})^{13}{ }^{14}$ is based on a subset of these parameters. The great majority of published ICD/CRT economic evaluations, however, including those informing NICE decisions, have been based on data or populations from single clinical trials. These analyses do not, therefore, reflect the totality of randomised controlled trial evidence available, and have only limited information with which to explore the potential for cost-effectiveness (and decisions based on cost-effectiveness) to vary by patient subgroups.

This paper reports a unique collaboration between manufacturers, clinicians and health economists to pool individual patient data from all major randomised controlled trials of these devices $(\mathrm{N}=12638)$. This database of studies has been used to inform a range of research studies. ${ }^{15} 16$ We report its use to develop a cost-effectiveness analysis for submission to NICE as part of their comprehensive review of ICD and CRT designed to answer the question 'in which patients are ICD/CRT-P/CRT-D cost-effective??. ${ }^{16}$ Unlike previous studies, the analysis is based on a synthesis of evidence across trials and reflects important differences between subgroups, hence guiding health systems' resource allocation decisions regarding these devices.

\section{METHODS}

\section{Decision analytical modelling}

The analysis is based on a typical UK HFrEF patient population, starting age of 66 years, all NYHA classes and LVEF $\leq 35 \%$. The costeffectiveness analysis follows the methods
Woods $B$, Hawkins $N$, et al. Heart 2016;102:1742-

1749. 
recommended by NICE. ${ }^{17}$ Costs considered are those of the UK National Health Service (NHS), and outcomes are expressed as quality-adjusted life-years (QALYs). An annual discount rate of $3.5 \%$ is applied to both costs and benefits. ${ }^{17}$

The analysis is based on a decision analytical model which comprises a series of regression equations to predict: mortality, hospitalisation rates and health-related quality of life (HRQoL). The regression equations include covariables representing patients' baseline prognostic characteristics. The first regression equation predicts the probability of death in patients who only receive medical therapy. This is combined with estimates of the treatment effects of ICD, CRT-P and CRT-D based on the results of a network meta-analyses ${ }^{15}$ to derive device-specific mortality probabilities. A second equation is used to predict the monthly probability of experiencing a hospitalisation event for any reason. A final equation estimates a patient's HRQoL given their characteristics and treatment. All living patients potentially incur other costs related to device implant and replacement, background medication and routine clinical visits.

Cost-effectiveness results are generated in a two-stage process. In the first stage, costs and QALYs are estimated for all interventions for all possible sets of patient characteristics ('patient profiles'). In the second, these are collapsed to 24 subgroups defined by NYHA class, QRS duration and presence or absence of LBBB. Due to clinical contraindications or a paucity of evidence, not all treatments are evaluated for each of the 24 subgroups (see online supplementary appendix 1, for details).

Expected (mean) costs and QALYs are estimated for all relevant treatments in each subgroup, and the following standard 'decision rules' are followed to identify the cost-effective intervention in each subgroup. ${ }^{18}$ First, any option that is less effective and more costly than one or more others is removed from consideration (dominated). Second, the extra cost per additional QALY (the incremental cost-effectiveness ratio (ICER)) of a more effective treatment is calculated and any treatment that is less effective than another with a higher ICER is removed from consideration (extended dominance). The remaining options lie on a cost-effectiveness 'frontier' which runs from least to most costly/effective. ICERs are calculated between each progressively more costly and effective option.

\section{Estimating model inputs}

The baseline mortality risk (patients receiving medical therapy alone) was estimated using relevant individual patient data from the included trials. ${ }^{15}$ Parametrical survival analysis was used to extrapolate these mortality risks beyond the follow-up periods in the trials in order to generate lifetime estimates. The following candidate baseline covariables were selected based on data availability, a review of risk scores, clinical guidelines, randomised controlled trial (RCT) subgroup analyses and clinical advice: age, QRS duration, LVEF, gender, NYHA class, ischaemic aetiology, LBBB status and a binary geographical indicator to track whether or not patients were from a North American centre. Final covariable selection in all regression models was via a stepwise procedure unless otherwise stated. Estimation of the treatment effects of the devices on all-cause mortality has been detailed elsewhere. ${ }^{15}$ Mortality treatment effects were assumed to be maintained for 7.5 years (the maximum follow-up period in the trials) after which they would decline so that, by 20 years, there was no impact on mortality.

The expected number of all-cause hospitalisations per month for patients on medical therapy, together with the treatment effects of the devices, was estimated from the 11 trials reporting relevant data (full list of studies reported in online supplementary appendix 2). Hospitalisation rates were assumed constant over a patient's lifetime. Excluding LBBB morphology, the covariates of interest were as for all-cause mortality. NYHA I-II patients were considered together due to paucity of data on NYHA I patients. For the analysis of the effect of devices on hospitalisation rates, study-specific intercepts were included as well as device-related main effects with interaction terms used to identify treatment effect modifiers.

HRQoL estimates expressed on a 0 (equivalent of dead) to 1 (equivalent of good health) scale are necessary to quality-adjust survival and calculate QALYs. ${ }^{18}$ Baseline HRQoL conditional upon patients' characteristics was estimated using data from the three trials reporting EuroQol-5Dimension (EQ-5D) data (relevant studies listed in online supplementary appendix 2). The treatment effects of devices are estimated as the change from baseline (to first follow-up) in the treatment arms of the included trials, minus the change from baseline in patients allocated to medical therapy. This HRQoL treatment effect is assumed to be maintained for 5 years based on information in the CArdiac REsynchronization - Heart Failure (CARE-HF) trial, ${ }^{19}$ and then to decline to 0 by 10 years. Throughout the model, a decline in HRQoL is applied to reflect ageing, estimated using UK general population data. ${ }^{20}$

Hospitalisation costs are based on information on hospitalisation type from a UK-based population study. ${ }^{21}$ The typical HF medications for each NYHA class are estimated based on a review of the clinical literature and expert opinion. With the exception of those relating to device systems, all costs are taken from national databases. ${ }^{22-24}$ The total implant cost for CRT-P is based on a relevant Healthcare Resource Group code. For CRT-D and ICD, no such codes exist, so cross-manufacturer average selling prices for both systems and leads were made available by the Association of British Healthcare Industries for the purpose of this analysis (see online supplementary appendix 3 ).

Device longevity estimates are based on data from the Central Cardiac Audit Database. Parametrical survival models are used to model time to first and subsequent device replacements (see online supplementary appendix 3).

A range of sensitivity and scenario analyses are undertaken to quantify the importance of the key modelling assumptions. Of particular importance is the uncertainty in assumptions regarding the duration of the mortality and HRQoL treatment effects. Alternative values explored as sensitivity analyses were: (1) a constant, lifetime mortality effect (as in previous UK reimbursement models ${ }^{10}{ }^{12}$ ); (2) a 5 year time period; and (3) the mean follow-up period from the studies included in the data analyses (2.54 years). A sensitivity analysis was also conducted using lifetime treatment effect durations for both all-cause mortality and HRQoL (as per previous UK reimbursement models). Further sensitivity analyses were undertaken including varying key costs, increasing device longevity, the use of an alternative approach to modelling all-cause hospitalisation and the use of NYHA class as a modifier of all-cause mortality treatment effect.

\section{Software}

All mortality related analyses were performed in R (http://www. r-project.org), with all analysis of hospitalisation and HRQoL performed in STATA V.12 (StataCorp. College Station, Texas, USA). The economic model was developed in Microsoft Excel (Microsoft, Redmond, Washington DC, USA).

\section{RESULTS}

\section{All-cause mortality}

Full details of the mortality model are reported in the supplementary appendix 4 . The risk of death was higher in patients 
who are older, male or with ischaemic aetiology. The risk of death was also found to increase across NYHA classes, and was more than three times higher in individuals in NYHA Class IV than those in NYHA I/II. The risk of mortality was lower in patients with a normal $(<120 \mathrm{~ms})$ versus longer QRS duration $(\mathrm{HR}=0.84)$ and decreased as LVEF increased. Long-term survival predictions for each subgroup and treatment are presented in the supplementary appendix 5. Allowing for variations in within-group sample size and covariate mix the results are broadly internally consistent and in line with the published literature.

\section{All-cause hospitalisation}

Full details of the baseline hospitalisation model are reported in the supplementary appendix 4. Patients in NYHA Classes III and IV were 2.1 times and 4.4 times, respectively, more likely to be hospitalised than those in NYHA Class I/II. Ischaemic aetiology increased the rate of hospitalisation by $9 \%$ and wide QRS complex increased the rate by $22 \%(120-149 \mathrm{~ms})$ and $6 \%$ (150 ms or more), respectively.

The model predicted that ICDs reduced monthly hospitalisation rates by $20 \%$ in patients with NYHA I/II/III HF. CRT-P was associated with reductions in monthly hospitalisation rates of $32 \%$ and $40 \%$, respectively, in patients with NYHA III/IV HF. CRT-D was associated with a monthly rate reduction of $30 \%$ in patients with NYHA I-IV HF. In NYHA III/IV patients the treatment effects arising from the patient level data for CRT-D compared with those generated for CRT-P were considered clinically implausible. For the base case analysis of these patients we therefore assumed equivalence of efficacy for CRT-D and
CRT-P. Subgroup/treatment-specific lifetime hospitalisation counts are reported in supplementary appendix 5 . These data require careful interpretation since subgroups with longer overall survival have more hospitalisation events.

\section{Health-related quality of life}

Full details of the HRQoL model are reported in the supplementary appendix 4. Statistically significant $(p<0.05)$ but modest improvements from baseline were observed for ICD and CRT-D in patients with NYHA I or II heart failure, and CRT-P in NYHA Class III $(+0.02,+0.03$ and +0.091 , respectively). No significant impact was observed on HRQoL with ICD or CRT-D therapy in patients in NYHA Class III. Meaningful results for patients with NYHA IV HF could not be generated due to the very low numbers of patients in the clinical trials.

For the purposes of economic modelling, equivalence of HRQoL benefit for CRT-P and CRT-D was again assumed in NYHA III and IV HF. This assumption can be justified on the basis of a review of external sources and trial-specific Minnesota Living with Heart Failure data. ${ }^{16}$

\section{Cost-effectiveness-base case}

The base case results for all subgroups are presented in table 1 . For each patient subgroup, the order of treatments on the costeffectiveness frontier is shown, and the ICERs for each option which is not subject to dominance or extended dominance. Table 2 shows the cost-effective option for each subgroup (costeffectiveness threshold: $£ 30000$ per QALY gained). Device therapy is cost-effective at this threshold in all subgroups examined. CRT-D is cost-effective in 10 of the 24 subgroups, and is

Table 1 Base case results. Cost-effectiveness sequence relates to the order in which interventions appear on the cost-effectiveness frontier

\begin{tabular}{|c|c|c|c|c|c|c|c|c|}
\hline \multirow[b]{2}{*}{ Patient group } & \multicolumn{4}{|c|}{ Cost-effectiveness sequence } & \multicolumn{4}{|c|}{ Incremental cost-effective ratios } \\
\hline & 1 st & 2nd & 3 rd & 4th & 1st & 2nd & 3 rd & 4th \\
\hline \multicolumn{9}{|l|}{ Individuals without LBBB morphology } \\
\hline NYHA I, QRS duration $<120 \mathrm{~ms}^{*}$ & MT & ICD & N/A & N/A & Referent & £24 074 & $\mathrm{~N} / \mathrm{A}$ & N/A \\
\hline NYHA I, QRS duration $\geq 120 \mathrm{~ms}$ and $<150 \mathrm{~ms}^{*}$ & MT & CRT-D & ICD & $\mathrm{N} / \mathrm{A}$ & Referent & Dominated & f16 253 & $\mathrm{~N} / \mathrm{A}$ \\
\hline NYHA I, QRS duration $\geq 150 \mathrm{~ms}^{*}$ & MT & ICD & CRT-D & $\mathrm{N} / \mathrm{A}$ & Referent & f21 102 & f21 759 & $\mathrm{~N} / \mathrm{A}$ \\
\hline NYHA II, QRS duration $<120 \mathrm{~ms}$ & MT & ICD & N/A & $\mathrm{N} / \mathrm{A}$ & Referent & f24 465 & $\mathrm{~N} / \mathrm{A}$ & $\mathrm{N} / \mathrm{A}$ \\
\hline NYHA II, QRS duration $\geq 120 \mathrm{~ms}$ and $<150 \mathrm{~ms}$ & MT & CRT-D & $I C D$ & N/A & Referent & Dominated & f16 813 & $\mathrm{~N} / \mathrm{A}$ \\
\hline NYHA II, QRS duration $\geq 150 \mathrm{~ms}$ & MT & ICD & CRT-D & $\mathrm{N} / \mathrm{A}$ & Referent & $f 20602$ & f23 738 & $\mathrm{~N} / \mathrm{A}$ \\
\hline NYHA III, QRS duration $<120 \mathrm{~ms}$ & MT & ICD & N/A & N/A & Referent & $£ 27826$ & N/A & $\mathrm{N} / \mathrm{A}$ \\
\hline NYHA III, QRS duration $\geq 120 \mathrm{~ms}$ and $<150 \mathrm{~ms}$ & MT & CRT-P & ICD & CRT-D & Referent & f20 178 & Ext Dominated & $\mathrm{f} 23349$ \\
\hline NYHA III, QRS duration $\geq 150 \mathrm{~ms}$ & MT & ICD & CRT-P & CRT-D & Referent & Dominated & f13 930 & f25 200 \\
\hline NYHA IV, QRS duration $<120 \mathrm{~ms}$ & MT & $\mathrm{N} / \mathrm{A}$ & $N / A$ & $N / A$ & Referent & N/A & $\mathrm{N} / \mathrm{A}$ & $N / A$ \\
\hline NYHA IV, QRS duration $\geq 120 \mathrm{~ms}$ and $<150 \mathrm{~ms}$ & MT & CRT-P & CRT-D & $\mathrm{N} / \mathrm{A}$ & Referent & f22 578 & f40 052 & $\mathrm{~N} / \mathrm{A}$ \\
\hline NYHA IV, QRS duration $\geq 150 \mathrm{~ms}$ & MT & CRT-P & CRT-D & N/A & Referent & f17 175 & f35 811 & $\mathrm{~N} / \mathrm{A}$ \\
\hline \multicolumn{9}{|l|}{ Individuals with LBBB morphology } \\
\hline NYHA I, QRS duration $\geq 120 \mathrm{~ms}$ and $<150 \mathrm{~ms}^{*}$ & MT & ICD & CRT-D & N/A & Referent & $£ 20677$ & f21 672 & $\mathrm{~N} / \mathrm{A}$ \\
\hline NYHA I, QRS duration $\geq 150 \mathrm{~ms}^{*}$ & MT & $I C D$ & CRT-D & $\mathrm{N} / \mathrm{A}$ & Referent & Ext Dominated & f17 470 & N/A \\
\hline NYHA II, QRS duration $\geq 120 \mathrm{~ms}$ and $<150 \mathrm{~ms}$ & MT & $I C D$ & CRT-D & N/A & Referent & Ext Dominated & f20 704 & $\mathrm{~N} / \mathrm{A}$ \\
\hline NYHA II, QRS duration $\geq 150 \mathrm{~ms}$ & MT & $I C D$ & CRT-D & $\mathrm{N} / \mathrm{A}$ & Referent & Ext Dominated & f17 664 & $\mathrm{~N} / \mathrm{A}$ \\
\hline NYHA III, QRS duration $\geq 120 \mathrm{~ms}$ and $<150 \mathrm{~ms}$ & MT & $I C D$ & CRT-P & CRT-D & Referent & Dominated & f14 215 & f24 875 \\
\hline NYHA III, QRS duration $\geq 150 \mathrm{~ms}$ & MT & $I C D$ & CRT-P & CRT-D & Referent & Dominated & f10 496 & $\mathrm{f} 28646$ \\
\hline NYHA IV, QRS duration $\geq 120 \mathrm{~ms}$ and $<150 \mathrm{~ms}$ & MT & CRT-P & CRT-D & $\mathrm{N} / \mathrm{A}$ & Referent & f18 664 & f37 104 & $\mathrm{~N} / \mathrm{A}$ \\
\hline NYHA IV, QRS duration $\geq 150 \mathrm{~ms}$ & MT & CRT-P & CRT-D & N/A & Referent & f14 500 & f40 449 & $\mathrm{~N} / \mathrm{A}$ \\
\hline
\end{tabular}


Table 2 Summary of cost-effectiveness recommendations arising from the base case analysis (threshold value: $£ 30000$ per QALY gained)

\begin{tabular}{|c|c|c|c|}
\hline NYHA & $\begin{array}{l}\text { QRS duration } \\
<120 \mathrm{~ms}\end{array}$ & $\begin{array}{l}\text { QRS duration } \\
120-150 \mathrm{~ms}\end{array}$ & $\begin{array}{l}\text { QRS duration } \\
>150 \mathrm{~ms}\end{array}$ \\
\hline \multicolumn{4}{|c|}{ Patients without LBBB morphology } \\
\hline $\mathrm{I}^{*}$ & $\mathrm{ICD}$ & ICD & CRT-D \\
\hline II & $\mathrm{ICD}$ & ICD & CRT-D \\
\hline III & $\mathrm{ICD}$ & CRT-P/CRT-D† & CRT-P/CRT-D $\dagger$ \\
\hline IV & Medical therapy & CRT-P & CRT-P \\
\hline \multicolumn{4}{|c|}{ Patients with LBBB morphology } \\
\hline$I^{*}$ & & CRT-D & CRT-D \\
\hline ॥ & & CRT-D & CRT-D \\
\hline III & & CRT-P/CRT-D $†$ & CRT-P/CRT-D† \\
\hline IV & & CRT-P & CRT-P \\
\hline \multicolumn{4}{|c|}{$\begin{array}{l}\text { *Results in NYHA I and IV patients are based on relatively low patient numbers and } \\
\text { may be subject to bias due to the nature of trial inclusion criteria for NYHA I } \\
\text { patients. For further detail see main text. } \\
\text { tInstances where NICE recommended two devices based on fully incremental results } \\
\text { and a threshold of f30 } 000 \text { per QALY gained. CRT-P values are relative to medical } \\
\text { therapy and CRT-D values relative to CRT-P. } \\
\text { CRT, cardiac resynchronisation therapy; CRT-D, combined CRT and ICD device; CRT-P, } \\
\text { stand-alone CRT device; ICD, implantable cardioverter defibrillator; LBBB, left bundle } \\
\text { branch block; NICE, National Institute for Health and Care Excellence; NYHA, } \\
\text { New York Heart Association; QALY, quality-adjusted life-year. }\end{array}$} \\
\hline
\end{tabular}

cost-effective for all LBBB morphology patients with NYHA I/II/III. ICDs are cost-effective for all non-NYHA IV patients with a QRS duration $<120 \mathrm{~ms}$ and for NYHA I/II, non-LBBB morphology patients with a QRS duration between $120 \mathrm{~ms}$ and 149 ms. CRT-P is cost-effective for all NYHA IV patients evaluated.

Results generated using a more stringent threshold value ( $£ 20000$ per QALY gained) are presented in table 3. CRT-D is only cost-effective in two patient groups (NYHA I or II, QRS duration $\geq 150 \mathrm{~ms}$, with $\mathrm{LBBB}$ ); ICD is only cost-effective in two patient groups (NYHA I or II, QRS duration between 120 $\mathrm{ms}$ and $149 \mathrm{~ms}$, no LBBB); and CRT-P is cost-effective in six patient groups (NYHA III or IV, QRS duration $>120 \mathrm{~ms}$, and LBBB and also NYHA III/IV, QRS duration $\geq 150 \mathrm{~ms}$ and no LBBB).

Table 3 Summary of cost-effectiveness recommendations arising from the base case analysis (threshold value: $£ 20000$ per QALY gained)

\begin{tabular}{clll}
\hline NYHA & $\begin{array}{l}\text { QRS duration } \\
<120 \mathrm{~ms}\end{array}$ & $\begin{array}{l}\text { QRS duration } \\
120-150 \mathrm{~ms}\end{array}$ & $\begin{array}{l}\text { QRS duration } \\
>150 \mathrm{~ms}\end{array}$ \\
\hline $\begin{array}{l}\text { Patients without } \\
\text { LBBB morphology }\end{array}$ & & \\
I* & Medical therapy & ICD & Medical therapy \\
III & Medical therapy & ICD & Medical therapy \\
IV & Medical therapy & Medical therapy & CRT-P \\
Patients with LBBB morphology & Medical therapy & Medical therapy & CRT-P \\
I* & & \\
II & & Medical therapy & CRT-D \\
III & Medical therapy & CRT-D \\
IV & & CRT-P & CRT-P \\
\hline
\end{tabular}

${ }^{*}$ Results in NYHA I and IV patients are based on relatively low patient numbers and may be subject to bias due to the nature of trial inclusion criteria for NYHA I patients. For further detail see main text.

CRT, cardiac resynchronisation therapy; CRT-D, combined CRT and ICD device; CRT-P, stand-alone CRT device; ICD, implantable cardioverter defibrillator; LBBB, left bundle branch block; NYHA, New York Heart Association; QALY, quality-adjusted life-year.
The nature of the cost-effective intervention is dependent upon the cost-effectiveness threshold (figure 1). Beyond a threshold value of approximately $£ 24500$ per QALY gained, for patients without LBBB morphology, the treatments identified in the base case analysis are largely robust to changes in threshold. For patients with LBBB morphology there is generally less sensitivity to changes in threshold, with the cost-effective treatments presented in table 2 largely unchanged beyond a threshold value of approximately $£ 22000$ per QALY.

Results in NYHA I and IV patients are subject to additional uncertainty due to the numbers of patients informing these analyses and the nature of the trial inclusion criteria for NYHA I patients, these issues are described in the discussion.

\section{Sensitivity analyses}

The choice of cost-effective therapy was, in general, unchanged when varying the duration of maximum all-cause mortality treatment effect (table 4, threshold value $£ 30000$ per QALY gained). A similar outcome was noted when the assumption of lifetime treatment effect durations for both all-cause mortality and HRQoL improvements was made (table 4). A detailed breakdown of the fully incremental ICERs generated in this latter analysis is presented in the supplementary appendix 6 . The corresponding results generated using a threshold value of $£ 20000$ per QALY gained are presented in table 5 .

The impact of varying key costs, increasing device longevity, the use of an alternative approach to modelling all-cause hospitalisation and the use of NYHA class as an all-cause mortality treatment effect modifier on the choice of devices at thresholds of $£ 20000$ and $£ 30000$ per QALY gained was modest. Hence, the model was robust to changes in these parameters.

\section{DISCUSSION}

The clinical efficacy of CRT and ICD therapy has been investigated in numerous studies, and these technologies are established therapies for HFrEF individuals in international practice. The uniqueness of the database created for the purpose of this analysis lies in the ability to explore the clinical efficacy of CRT and ICD in detail, and in particular to pursue a thorough investigation of the impact of key clinical variables on both the baseline risk of death and the efficacy of each treatment option in reducing mortality. ${ }^{15}$ The database also facilitated the incorporation of clinical subgroups into the cost-effectiveness assessment so the devices representing best value for money for patients with different sets of clinical characteristics could be identified.

This collaboration can serve as a model for similar collaboration across manufacturers. Pooling together clinical data across manufactures can help manufactures, physicians and policy makers become more confident in the clinical-effectiveness and cost-effectiveness of a therapy and get a better understanding of how this varies across patients.

The analysis presented here was used to inform the recent NICE guidance regarding the use of these technologies. The committee developing the NICE recommendations recognised this analysis as 'a rich and important data source', 25 and based its decision making on it. The guidance issued by NICE in 2014 reflected the findings of this cost-effectiveness analysis in almost all subgroups, ${ }^{25}$ with the exception of CRT-D in patients with NYHA III and QRS 120-149 ms without LBBB morphology and CRT-D in asymptomatic (NYHA Class I) patients with QRS 120-149 ms. ICD was recommended in both cases due to concerns about reduced effectiveness and a lack of symptomatic benefit of CRT in these subgroups. 

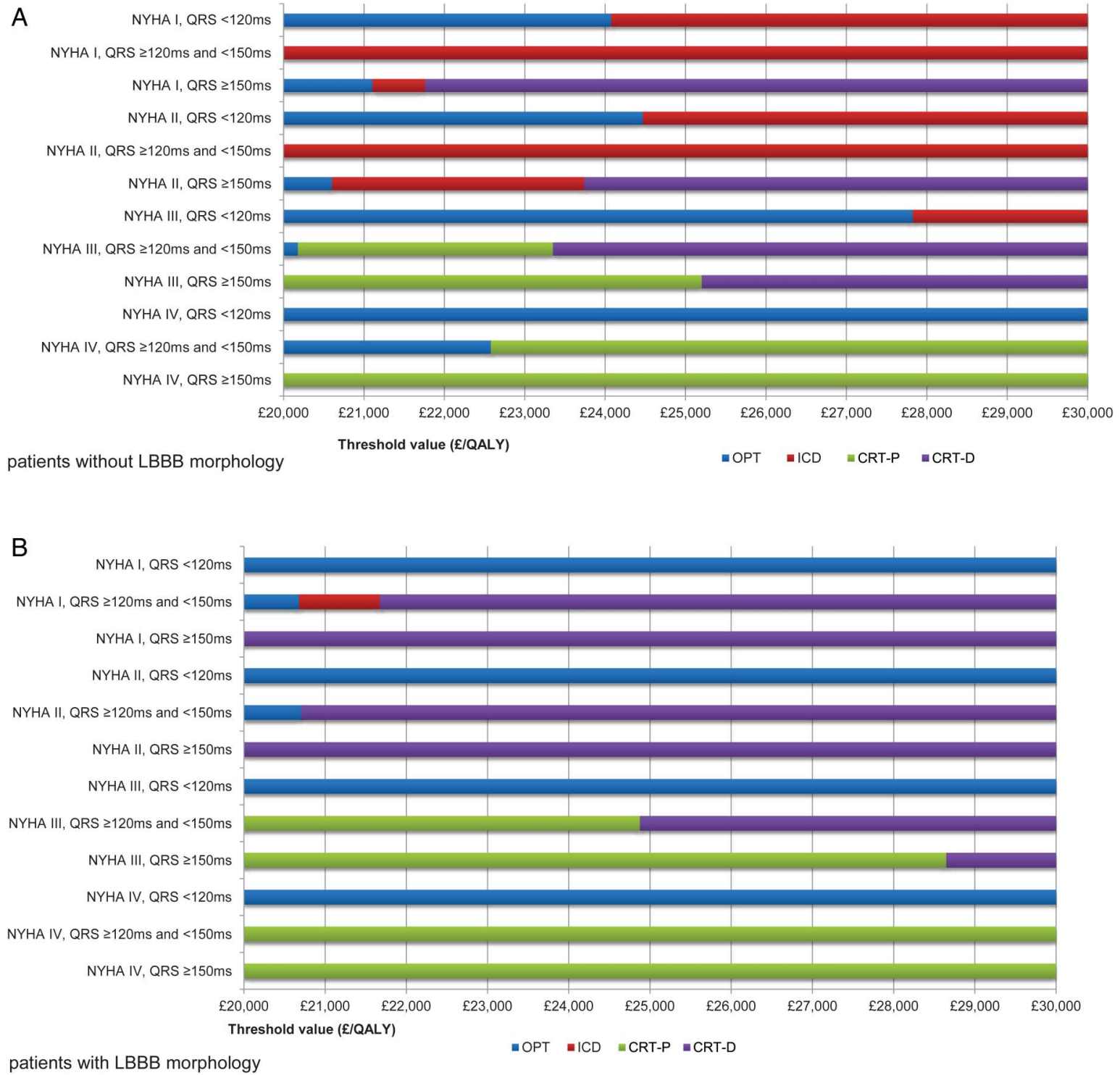

Figure 1 Graphic display of cost-effective options across cost-effectiveness threshold values (base case). CRT, cardiac resynchronisation therapy; CRT-D, combined CRT and ICD device; CRT-P, stand-alone CRT device; ICD, implantable cardioverter defibrillator; LBBB, left bundle branch block; NYHA, New York Heart Association; QALY, quality-adjusted life-year.

The major source of uncertainty identified during the NICE appraisal of these technologies was the duration of treatment effect on all-cause mortality. The duration of effect used in this analysis (7.5 years) is supported by a number of long-term studies. Recently published data from the CARE-HF study ${ }^{19}$ found that, at a mean follow-up of 56 months in the CRT-P and 50 months in the medical therapy arms, the HR for all-cause mortality (CRT-P vs optimal pharmacological therapy (OPT)) was 0.77 (95\% CI 0.63 to 0.93 ), despite $39 \%$ of control patients crossing over to a CRT device during follow-up. In addition, long-term follow-up from the Multicenter Automatic Defibrillator Implantation Trial-II (MADIT-II) $\mathrm{study}^{26}$ found that, at a median follow-up of 7.6 years, the HR for all-cause mortality (ICD vs non-ICD) was 0.77 (0.65 to 0.91 ), although $34 \%$ of control patients crossed over to a device during follow-up. Attempts to adjust statistically for cross-over resulted in treatment effect estimates of 0.67 (0.56 to 0.80$)$ and 0.66 (0.56 to 0.78), in CARE-HF and MADIT-II, respectively. ${ }^{19} 26$

The results from our analysis, generated using a costeffectiveness threshold of $£ 30000$ per QALY gained, broadly agrees with guidelines issued by the European Society of Cardiology. ${ }^{3}{ }^{27}$ In patients with QRS $<120 \mathrm{~ms}$, NYHA II-III and ejection fraction $(\mathrm{EF}) \leq 35 \%$, ICD is recommended and costeffective according to our analysis. In ambulatory NYHA IV patients with prolonged QRS duration, CRT-P is recommended and cost-effective. Our analysis provides information on where scarce resources should be targeted in patients with QRS prolongation and in NYHA Class II or III where several possible device options are recommended. In particular, it suggests that CRT-D is cost-effective in NYHA II-III patients with LBBB morphology, and that, in patients with non-LBBB morphology, CRT-D is cost-effective in all groups with the exception of NYHA II patients with a QRS duration of 120-149 ms. Unlike previous guidelines, the current analysis suggests implantable devices may be cost-effective in asymptomatic patients (NYHA Class I). However, given the small number of patients in these groups in the trials, results in NYHA Class I, and in particular in relation to CRT-D in this patient group, should be regarded with caution. ${ }^{15}$

Comparison of the results from the current analysis with previous UK Health Technology Assessment (HTA) models of ICD 
Table 4 Impact of duration of treatment effect on treatment choice (threshold $f 30000$ per QALY gained)

\begin{tabular}{|c|c|c|c|c|c|}
\hline \multirow[b]{2}{*}{ Subgroup } & \multicolumn{4}{|c|}{ Duration of mortality treatment effect } & \multirow{2}{*}{$\begin{array}{l}\text { Lifetime mortality } \\
\text { and constant HRQoL }\end{array}$} \\
\hline & Lifetime & 7.5 years & 5 years & Mean f/up & \\
\hline \multicolumn{6}{|l|}{ Individuals without LBBB } \\
\hline NYHA I, QRS $<120 \mathrm{~ms}^{*}$ & $I C D$ & ICD & $I C D$ & $I C D$ & $I C D$ \\
\hline NYHA I, QRS $\geq 120 \mathrm{~ms}$ and $<150 \mathrm{~ms}^{*}$ & $I C D$ & ICD & ICD & $I C D$ & $I C D$ \\
\hline NYHA I, QRS $\geq 150 \mathrm{~ms}^{*}$ & CRT-D & CRT-D & CRT-D & CRT-D & CRT-D \\
\hline NYHA II, QRS $<120 \mathrm{~ms}$ & ICD & $I C D$ & $I C D$ & ICD & $I C D$ \\
\hline NYHA II, QRS $\geq 120 \mathrm{~ms}$ and $<150 \mathrm{~ms}$ & $I C D$ & $I C D$ & $I C D$ & $I C D$ & $I C D$ \\
\hline NYHA II, QRS $\geq 150 \mathrm{~ms}$ & CRT-D & CRT-D & CRT-D & CRT-D & CRT-D \\
\hline NYHA III, QRS <120 ms & ICD & ICD & ICD & MT & ICD \\
\hline NYHA III, QRS $\geq 120 \mathrm{~ms}$ and $<150 \mathrm{~ms}$ & CRT-D & CRT-D & CRT-D & CRT-D & CRT-D \\
\hline NYHA III, QRS $\geq 150 \mathrm{~ms}$ & CRT-D & CRT-D & CRT-D & CRT-D & CRT-D \\
\hline NYHA IV, QRS $<120 \mathrm{~ms}$ & MT & MT & MT & MT & MT \\
\hline NYHA IV, QRS $\geq 120 \mathrm{~ms}$ and $<150 \mathrm{~ms}$ & CRT-P & CRT-P & CRT-P & CRT-P & CRT-P \\
\hline NYHA IV, QRS $\geq 150 \mathrm{~ms}$ & CRT-P & CRT-P & CRT-P & CRT-P & CRT-P \\
\hline \multicolumn{6}{|l|}{ Individuals with LBBB } \\
\hline NYHA I, QRS $\geq 120 \mathrm{~ms}$ and $<150 \mathrm{~ms}^{*}$ & CRT-D & CRT-D & CRT-D & CRT-D & CRT-D \\
\hline NYHA I, QRS $\geq 150 \mathrm{~ms}^{*}$ & CRT-D & CRT-D & CRT-D & CRT-D & CRT-D \\
\hline NYHA II, QRS $\geq 120 \mathrm{~ms}$ and $<150 \mathrm{~ms}$ & CRT-D & CRT-D & CRT-D & CRT-D & CRT-D \\
\hline NYHA II, QRS $\geq 150 \mathrm{~ms}$ & CRT-D & CRT-D & CRT-D & CRT-D & CRT-D \\
\hline NYHA III, QRS $\geq 120 \mathrm{~ms}$ and $<150 \mathrm{~ms}$ & CRT-D & CRT-D & CRT-D & CRT-D & CRT-D \\
\hline NYHA III, QRS $\geq 150 \mathrm{~ms}$ & CRT-D & CRT-D & CRT-P & CRT-P & CRT-D \\
\hline NYHA IV, QRS $\geq 120 \mathrm{~ms}$ and $<150 \mathrm{~ms}$ & CRT-P & CRT-P & CRT-P & CRT-P & CRT-P \\
\hline NYHA IV, QRS $\geq 150 \mathrm{~ms}$ & CRT-P & CRT-P & CRT-P & CRT-P & CRT-P \\
\hline
\end{tabular}

Changes from base case highlighted in bold.

* Results in NYHA I and IV patients are based on relatively low patient numbers and may be subject to bias due to the nature of trial inclusion criteria for NYHA I patients. For further detail see main text.

CRT, cardiac resynchronisation therapy; CRT-D, combined CRT and ICD device; CRT-P, stand-alone CRT device; HRQoL, health-related quality of life; ICD, implantable cardioverter defibrillator; LBBB, left bundle branch block; MT, medical therapy; NYHA, New York Heart Association; QALY, quality-adjusted life-year.

Table 5 Impact of duration of treatment effect on treatment choice (threshold $f 20000$ per QALY gained)

\begin{tabular}{|c|c|c|c|c|c|}
\hline \multirow[b]{2}{*}{ Subgroup } & \multicolumn{4}{|c|}{ Duration of mortality treatment effect } & \multirow{2}{*}{$\begin{array}{l}\text { Lifetime mortality } \\
\text { and constant HRQoL }\end{array}$} \\
\hline & Lifetime & 7.5 years & 5 years & Mean f/up & \\
\hline \multicolumn{6}{|l|}{ Individuals without LBBB } \\
\hline NYHA I, QRS $<120 \mathrm{~ms}^{*}$ & $\mathrm{ICD}$ & MT & MT & MT & ICD \\
\hline NYHA I, QRS $\geq 120 \mathrm{~ms}$ and $<150 \mathrm{~ms}^{*}$ & $\mathrm{ICD}$ & $I C D$ & ICD & ICD & ICD \\
\hline NYHA I, QRS $\geq 150 \mathrm{~ms}^{*}$ & CRT-D & MT & MT & MT & CRT-D \\
\hline NYHA II, QRS <120 ms & $I C D$ & MT & MT & MT & ICD \\
\hline NYHA II, QRS $\geq 120 \mathrm{~ms}$ and $<150 \mathrm{~ms}$ & $I C D$ & $I C D$ & $I C D$ & ICD & $I C D$ \\
\hline NYHA II, QRS $\geq 150 \mathrm{~ms}$ & CRT-D & MT & MT & MT & ICD \\
\hline NYHA III, QRS $<120 \mathrm{~ms}$ & MT & MT & MT & MT & MT \\
\hline NYHA III, QRS $\geq 120 \mathrm{~ms}$ and $<150 \mathrm{~ms}$ & CRT-P & MT & MT & MT & CRT-P \\
\hline NYHA III, QRS $\geq 150 \mathrm{~ms}$ & CRT-P & CRT-P & CRT-P & CRT-P & CRT-P \\
\hline NYHA IV, QRS $<120 \mathrm{~ms}$ & MT & MT & MT & MT & MT \\
\hline NYHA IV, QRS $\geq 120 \mathrm{~ms}$ and $<150 \mathrm{~ms}$ & MT & MT & MT & MT & MT \\
\hline NYHA IV, QRS $\geq 150 \mathrm{~ms}$ & CRT-P & CRT-P & CRT-P & CRT-P & CRT-P \\
\hline \multicolumn{6}{|l|}{ Individuals with LBBB } \\
\hline NYHA I, QRS $\geq 120 \mathrm{~ms}$ and $<150 \mathrm{~ms}^{*}$ & CRT-D & MT & MT & MT & CRT-D \\
\hline NYHA I, QRS $\geq 150 \mathrm{~ms}^{*}$ & CRT-D & CRT-D & CRT-D & MT & CRT-D \\
\hline NYHA II, QRS $\geq 120 \mathrm{~ms}$ and $<150 \mathrm{~ms}$ & CRT-D & MT & MT & MT & CRT-D \\
\hline NYHA II, QRS $\geq 150 \mathrm{~ms}$ & CRT-D & CRT-D & CRT-D & MT & CRT-D \\
\hline NYHA III, QRS $\geq 120 \mathrm{~ms}$ and $<150 \mathrm{~ms}$ & CRT-P & CRT-P & CRT-P & CRT-P & CRT-P \\
\hline NYHA III, QRS $\geq 150 \mathrm{~ms}$ & CRT-P & CRT-P & CRT-P & CRT-P & CRT-P \\
\hline NYHA IV, QRS $\geq 120 \mathrm{~ms}$ and $<150 \mathrm{~ms}$ & CRT-P & CRT-P & CRT-P & CRT-P & CRT-P \\
\hline NYHA IV, QRS $\geq 150 \mathrm{~ms}$ & CRT-P & CRT-P & CRT-P & CRT-P & CRT-P \\
\hline
\end{tabular}

Changes from base case highlighted in bold.

${ }^{*}$ Results in NYHA I and IV patients are based on relatively low patient numbers and may be subject to bias due to the nature of trial inclusion criteria for NYHA I patients. For further detail see main text.

CRT, cardiac resynchronisation therapy; CRT-D, combined CRT and ICD device; CRT-P, stand-alone CRT device; HRQoL, health-related quality of life; ICD, implantable cardioverter defibrillator; LBBB, left bundle branch block; MT, medical therapy; NYHA, New York Heart Association; QALY, quality-adjusted life-year. 


\section{Key messages}

What is already known on this subject?

- Clinical guidelines on implantable cardioverter defibrillators (ICDs)/ cardiac resynchronisation therapy (CRT) in HF make recommendations for ICD, CRT pacemakers (CRT-P) and the combined device (CRT-D) based on a range of clinical parameters. These include New York Heart Association (NYHA) class, left ventricular ejection fraction, ischaemic aetiology status, QRS duration, and presence or absence of either atrial fibrillation or left bundle branch block (LBBB). ICD and CRT therapy are not indicated in all patient groups of interest.

- ICD, CRT-P and CRT-D have been shown to represent a cost-effective use of UK healthcare expenditure when compared with medical therapy, and when evaluated in patient groups determined by individual trial inclusion criteria.

\section{What might this study add?}

- We have compared the devices to each other, as well as no device therapy in a much wider set of patients than has previously been evaluated in order to provide an answer to the question 'in which patients are ICD/CRT-P/CRT-D cost-effective?'.

- Data from multiple RCTs rather than single studies were used to inform the cost-effectiveness analysis hence results are more reflective of the totality of the clinical data. Results are stratified by a series of commonly used clinical parameters and present healthcare decision makers with much more information than was previously available.

- Device therapy is cost-effective at a threshold of $£ 30000$ per quality-adjusted life-year gained in all subgroups examined. CRT-D is cost-effective in 10 of the 24 subgroups, and is cost-effective for all LBBB morphology patients with NYHA I-III. ICDs are cost-effective for all NYHA I-III patients with a QRS duration $<120 \mathrm{~ms}$ and for NYHA I-II patients with non-LBBB morphology and a QRS duration between $120 \mathrm{~ms}$ and $149 \mathrm{~ms}$. CRT-P is cost-effective for all NYHA IV patients evaluated.

\section{How might this impact on clinical practice?}

- The results from our work will allow healthcare decision makers to make more informed decisions on which devices to offer patients taking into account both clinical and economic factors.

and CRT is challenging due to the different model structures and evidence used. The earlier models used aggregate level evidence, whereas the current model was based on access to a large amount of individual patient data. Accepting these differences, the results from the sensitivity analysis performed with the removal of all tapering effects on treatment effect durations are the most comparable with earlier models. This showed that the ICERs from the current analysis are lower (ie, better value for money) than those considered acceptable in previous NICE guidance. The reasons for this are likely to be increases in average device longevity and a reduction in hardware acquisition costs. In many cases, where ICD was historically recommended, the current analysis suggests patients should be offered a CRT-D device as the most clinically effective and cost-effective option.

A number of limitations arose from the choice of modelling approach and data on which the analyses were based, with the main area of potential concern being that some of the patient groups modelled were sparsely represented in our database. The primary groups of concern relate to patients in NYHA Classes I and IV heart failure (HF) (regardless of LBBB status). Cost-effectiveness results in these groups may have been influenced by the small patient numbers and should be treated with caution. In addition, NYHA I patients in trials may be atypical of those observed in clinical practice as specific inclusion criteria were used to focus on patients who were easily identifiable. ${ }^{28} 29$

Omitting previous HF hospitalisations as a predictor of subsequent monthly hospitalisation events represents a limitation of our analysis. However, the total event rates predicted are low and the choice of modelling approach is therefore unlikely to have had a substantive impact on the model results.

In conclusion, from a UK NHS perspective, at a threshold of $£ 30000$ per QALY gained our analysis has shown that CRT-D is cost-effective in a far wider group of patients than previously recommended and that, for most other patients, ICD is a costeffective treatment alternative. Our analysis also showed that CRT$\mathrm{P}$ was cost-effective in all patients with NYHA III/IV and a QRS duration $>120 \mathrm{~ms}$. Device therapy is cost-effective in most patient groups with LBBB at a threshold of $£ 20000$ per QALY gained.

Acknowledgements The authors thank Dr David Cunningham (on behalf of the Central Cardiac Audit Database (CCAD)) for making the device longevity data available (see online supplementary appendix 3 ) and to the device manufacturers for making available all relevant trial data.

Funding This work was supported by Biotronik, Boston Scientific, Medtronic, Sorin and St. Jude Medical. MRC's salary is funded by the National Institute for Health Research Cardiovascular Biomedical Research Unit at the Royal Brompton Hospital, London. CJP's salary is funded by Newcastle upon Tyne Hospitals NHS Foundation Trust.

Competing interests $\mathrm{BW}, \mathrm{SM}$, and $\mathrm{NH}$ were employees of ICON during the period of this work. MS is a consultant to ICON. and received payment for his involvement in this work. MRC provides consultancy advice to Medtronic, Boston Scientific and St. Jude Medical and has had research grants from Medtronic. CJP has received research funding and travel grants to attend scientific meetings and has provided consultancy advice to Medtronic, Boston Scientific and St. Jude Medical. HK has provided consultancy advice and has received research funding from Boston Scientific. WA has received consulting fees from St. Jude Medical and Biotronik.

Provenance and peer review Not commissioned; externally peer reviewed.

Open Access This is an Open Access article distributed in accordance with the Creative Commons Attribution Non Commercial (CC BY-NC 4.0) license, which permits others to distribute, remix, adapt, build upon this work non-commercially, and license their derivative works on different terms, provided the original work is properly cited and the use is non-commercial. See: http://creativecommons.org/ licenses/by-nc/4.0/

\section{REFERENCES}

1 Hunt SA, Abraham WT, Chin MH, et al. ACC/AHA 2005 Guideline Update for the Diagnosis and Management of Chronic Heart Failure in the Adult: a report of the American College of Cardiology/American Heart Association Task Force on Practice Guidelines (Writing Committee to Update the 2001 Guidelines for the Evaluation and Management of Heart Failure): developed in collaboration with the American College of Chest Physicians and the International Society for Heart and Lung Transplantation: endorsed by the Heart Rhythm Society. Circulation 2005;112:e154-235.

2 Jessup M, Abraham WT, Casey DE, et al. 2009 focused update: ACCF/AHA Guidelines for the Diagnosis and Management of Heart Failure in Adults: a report of the American College of Cardiology Foundation/American Heart Association Task Force on Practice Guidelines: developed in collaboration with the International Society for Heart and Lung Transplantation. Circulation 2009;119:1977-2016.

3 McMurray JJ, Adamopoulos S, Anker SD, et al. ESC guidelines for the diagnosis and treatment of acute and chronic heart failure 2012: The Task Force for the Diagnosis and Treatment of Acute and Chronic Heart Failure 2012 of the European Society of Cardiology. Developed in collaboration with the Heart Failure Association (HFA) of the ESC. Eur J Heart Fail 2012;14:803-69. 
4 Noyes K, Veazie P, Hall WJ, et al. Cost-effectiveness of cardiac resynchronization therapy in the MADIT-CRT trial. J Cardiovasc Electrophysiol 2013;24:66-74.

5 Linde C, Mealing S, Hawkins N, et al. Cost-effectiveness of cardiac resynchronization therapy in patients with asymptomatic to mild heart failure: insights from the European cohort of the REVERSE (Resynchronization Reverses remodeling in Systolic Left Ventricular Dysfunction). Eur Heart J 2011;32:1631-9.

6 Mark DB, Nelson CL, Anstrom KJ, et al. Cost-effectiveness of defibrillator therapy or amiodarone in chronic stable heart failure: results from the Sudden Cardiac Death in Heart Failure Trial (SCD-HeFT). Circulation 2006;114:135-42.

7 Al-Khatib SM, Anstrom KJ, Eisenstein EL, et al. Clinical and economic implications of the Multicenter Automatic Defibrillator Implantation Trial-II. Ann Intern Med 2005:142:593-600.

8 Calvert MJ, Freemantle N, Yao G, et al. Cost-effectiveness of cardiac resynchronization therapy: results from the CARE-HF trial. Eur Heart J 2005;26:2681-8.

9 Feldman AM, de Lissovoy G, Bristow MR, et al. Cost effectiveness of cardiac resynchronization therapy in the Comparison of Medical Therapy, Pacing, and Defibrillation in Heart Failure (COMPANION) trial. J Am Coll Cardio/ 2005;46:2311-21.

10 Fox M, Mealing S, Anderson R, et al. The clinical effectiveness and cost-effectiveness of cardiac resynchronisation (biventricular pacing) for heart failure: systematic review and economic model. Health Technol Assess 2007; 11:iii-iv, ix-248.

11 Medical Services Advisory Committee Australian Government Department of Health and Aging. Cardiac resynchronisation therapy for severe heart failure. 2006. http:// www.msac.gov.au/internet/msac/publishing.nsf/Content/1FBFC70B8B62BA64 CA2575AD0082FDA4/\$File/1042-One_page_summary.pdf (accessed 9 Jun 2016).

12 Buxton $\mathrm{M}$, Caine N, Chase D, et al. A review of the evidence on the effects and costs of implantable cardioverter defibrillator therapy in different patient groups, and the modelling of cost-effectiveness and cost-utility for these groups in a UK context. Health Technol Assess 2006;10:iii-iv, ix-xi, 1-164.

13 National Institute for Health and Clinical Excellence. Implantable Cardioverter Defibrillators for Arrhythmias: Final Appraisal Determination. http://www.nice.org.uk/ nicemedia/pdf/Arrhythmias_FAD.pdf

14 National Institute for Health and Clinical Excellence. Cardiac resynchronisation therapy for the treatment of heart failure: Final Appraisal Determination. http://www.nice.org.uk/nicemedia/pdf/Heartfailure_FAD.pdf

15 Woods B, Hawkins N, Mealing S, et al. Individual patient data network metaanalysis of mortality effects of implantable cardiac devices. Heart 2015;101:1800-6.

16 Association of British Healthcare Industries. Implantable cardioverter defibrillators for the treatment of arrhythmias and cardiac resynchronisation therapy for the treatment of heart failure (review of TA95 and TA120): Submission to The National Institute for Health and Care Excellence. https://www.nice.org.uk/guidance/ta314/ documents

17 National Institute for Health and Care Excellence. Guide to the Methods of Technology Appraisal. https://www.nice.org.uk/article/pmg9/chapter/foreword

18 Briggs ACK, Sculpher M. Decision Modelling for Health Economic Evaluation. Oxford University Press; 2006

19 Cleland JG, Freemantle N, Erdmann E, et al. Long-term mortality with cardiac resynchronization therapy in the Cardiac Resynchronization-Heart Failure (CARE-HF) trial. Eur J Heart Fail 2012;14:628-34.

20 Kind PHG, Macran S. UK Population Norms for EQ-5D. https://www.york.ac.uk/ media/che/documents/papers/discussionpapers/CHE\%20Discussion\%20Paper\% 20172.pdf

21 Cowie MR, Fox KF, Wood DA, et al. Hospitalization of patients with heart failure: a population-based study. Eur Heart J 2002;23:877-85.

22 Department of Health. NHS Reference costs 2012. https://www.gov.uk/government/ collections/nhs-reference-costs

23 Curtis L. Unit costs of health and social care 2012 http://www.pssru.ac.uk/

24 British National Formulary. http://www.bnf.org/

25 National Institute for Health and Care Excellence. Implantable cardioverter defibrillators and cardiac resynchronisation therapy for arrhythmias and heart failure (review of TA95 and TA120): http://www.nice.org.uk/guidance/ta314/documents/ arrythmias-icds-heart-failure-cardiac-resynchronisation-fad-document2

26 Goldenberg I, Gillespie J, Moss AJ, et al. Long-term benefit of primary prevention with an implantable cardioverter-defibrillator: an extended 8-year follow-up study of the Multicenter Automatic Defibrillator Implantation Trial II. Circulation 2010;122:1265-71.

27 Brignole M, Auricchio A, Baron-Esquivias G, et al., The Task Force on cardiac pacing and resynchronization therapy of the European Society of Cardiology (ESC). Developed in collaboration with the European Heart Rhythm Association (EHRA). 2013 ESC Guidelines on cardiac pacing and cardiac resynchronization therapy. Rev Esp Cardiol (Engl Ed) 2014;67:58.

28 Linde C, Abraham WT, Gold MR, et al. Randomized trial of cardiac resynchronization in mildly symptomatic heart failure patients and in asymptomatic patients with left ventricular dysfunction and previous heart failure symptoms. J Am Coll Cardiol 2008;52:1834-43.

29 Moss AJ, Hall WJ, Cannom DS, et al. Cardiac-resynchronization therapy for the prevention of heart-failure events. N Engl J Med 2009;361:1329-38. 